\title{
Wildland Fire Management under COVID-19. Brief 1, Review of Materials
}

Peter Moore, Food and Agriculture Organization of the United Nations, Rome, Italy

Bethany Hannah, The American Wildfire Experience, USA

Jasper de Vries, Strategic Communication Group, Wageningen University, The Netherlands

Marijn Poortvliet, Strategic Communication Group, Wageningen University, The Netherlands

Ron Steffens, Wildfire Magazine, International Association of Wildland Fire; Prescott College, USA

Cathelijne R. Stoof, Department of Environmental Sciences, Wageningen University, The Netherlands

\section{Executive summary}

COVID-19 will have major implications for wildland fire management, because of severe social distancing and hygiene requirements. We collected procedures and guidance created around the world to help prepare wildland fire professionals globally for fire management during this pandemic. We did this by reviewing materials and sending out a survey to the fire community. We publish our findings in three ways. This Brief summarizes the materials reviewed and to give initial considerations and guidelines for the global wildland fire community. A follow up Brief will discuss the results of the survey, which will remain open until 15 May 2020 (link). Finally, results will be submitted to an academic journal for rapid publication. By using existing groups and networks to discuss this issue, obtain input and collate ideas, it will be possible to more quickly establish the principles and options for adapting to the constraints and opportunities that COVID-19 is imposing. All materials will be available free of charge for use by developed and developing countries, to facilitate learning and collaboration amongst individuals, agencies, and countries.

Please cite this report as: Moore, P., Hannah, B., de Vries, J., Poortvliet, M., Steffens, R., Stoof, C.R. (2020). Wildland Fire Management under COVID-19. Brief 1, Review of Materials. Wageningen University, The Netherlands. https://doi.org/10.18174/521344.

Corresponding authors: Peter.Moore@fao.org; Cathelijne.Stoof@wur.nl

This work is licensed under a Creative Commons Attribution 4.0 International License. This allows the user to redistribute, to create derivatives, such as a translation, and even use the publication for commercial activities, provided that appropriate credit is given to the author (BY) and that the user indicates whether the publication has been changed. For the license terms, see here. 


\section{Table of Contents}

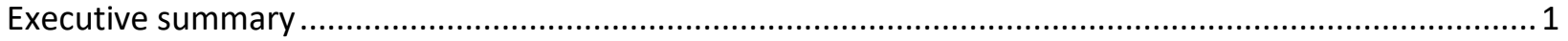

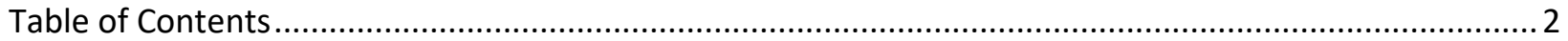

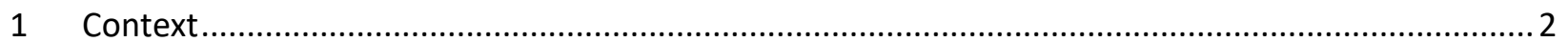

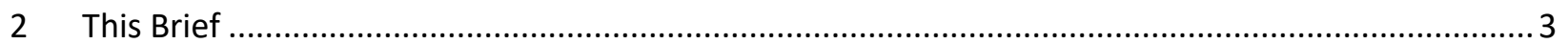

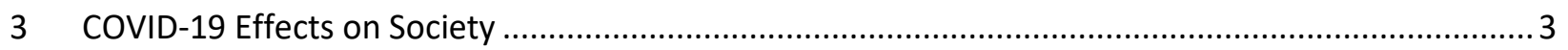

4 The Material Reviewed ……………………………...................................................

5 Fire Management Considerations..................................................................................... 4

$5.1 \quad$ Approaches to Fire Suppression …………………………………………………..

5.2 COVID-19 Limitations for Fire Management......................................................................

6 Initial Guidance for Fire Management under COVID-19..............................................................

6.1 General Principles and Approaches ..............................................................................

6.1.1 Keeping Firefighters Healthy to Reduce Risk of Contracting COVID-19 …........................ 5

6.1.2 Social Distancing ..............................................................................................

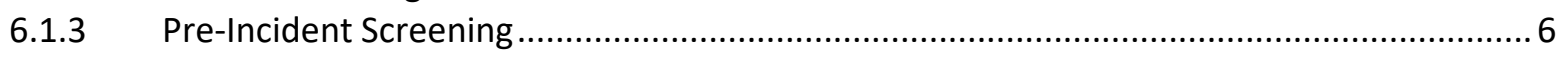

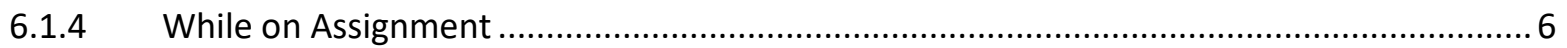

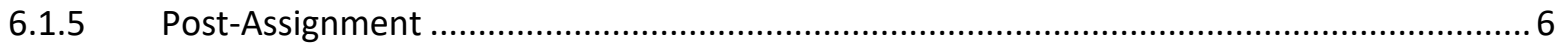

6.1.6 Transportation and Aviation ....................................................................................

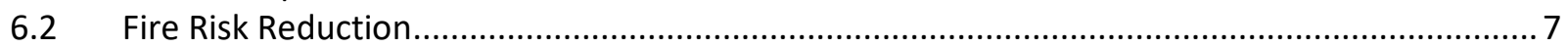

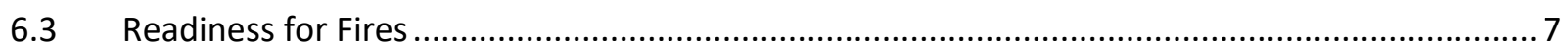

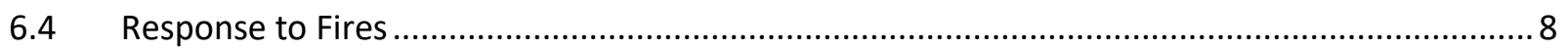

6.5 Protecting Remote Communities from Infection...............................................................

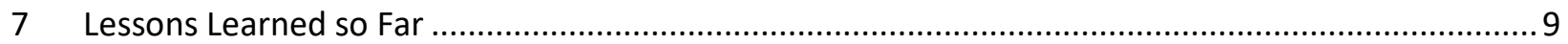

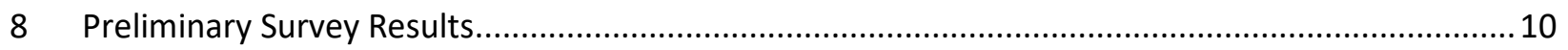

8.1 About the Survey: Fire Management Under COVID-19 ………………………………....... 10

8.2 Type of Responses to Date.................................................................................................. 10

8.3 Next Steps: Survey Still Open, Findings to be Published Late May 2020...................................12

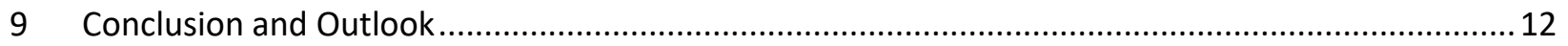

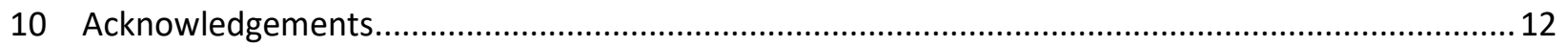

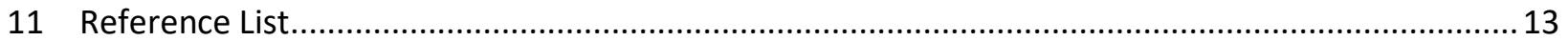

\section{Context}

As the Northern Hemisphere enters its summer, and the fire season, the requirements that have been put in place to deal with COVID-19 must be considered, and fire management procedures, standards and approaches need to be adapted. Past practice and 'normal' will need to be reviewed and probably be adjusted for this fire season, which has already started in NW Europe.

The focus of this Brief is on wildland fire suppression, but in time the full range of fire management should be considered as well, given the expected longevity of COVID-19 impacts. For that reason, it will be critical to continue incorporating COVID-19 requirements beyond the Northern Hemisphere summer, as this preliminary review already shows that COVID-19 affects all wildland fire management operations, including risk reduction. 


\section{This Brief}

We compiled this brief from materials sourced from agencies, provided by colleagues and submitted by individuals who completed an online survey distributed through Wageningen University. The goal of the survey was to compile materials, clarify the implications of COVID-19 restrictions on wildland fire management, map current thinking and raise awareness of this issue. Initial results of the survey are also reported here.

The intention was to compile and synthesise considerations and advice that have been drafted or formulated. The goal of this synthesis was to assist agencies and countries where preparation for fire management under COVID-19 has not yet been done, by suggesting strategies and tactics for fire management that could potentially be applied under the conditions required by the COVID-19 pandemic. The strategies and tactics have barely begun to be 'refined' or 'changed' through experience. As the summer progresses and operations are conducted, we plan to collect further insights, changes and adjustments to be incorporated, summarized and circulated as appropriate.

\section{COVID-19 Effects on Society}

The outbreak of Coronavirus Disease 2019 (COVID-19) led many countries around the world to declare a state of emergency. In most countries some level of restriction on movement, business and social interaction has been implemented. In many countries this has taken the form of a "lockdown"; nonessential businesses and industry sectors have been suspended in many countries. Efforts put in place to deal with COVID-19 include:

- Social distancing is to be practiced by all, including in work places that are allowed to remain open. The distance specified ranges from 1-2 metres.

- Gatherings of people are not permitted, with group sizes limited to a maximum of two and in some countries up to ten.

- People are required to remain indoors, except for essential tasks - obtaining food, medical appointments, exercise.

- Schools, universities and day care centres are physically closed.

Most countries have closed their borders and are not permitting travelers in or out and, in some cases, not allowing transit. In many countries, travel within the nation (between regions, states or provinces) is also restricted, for example in Italy. As countries begin to consider easing restrictions, many are increasing their capacity to track and trace infected people and monitor the population while continuing to improve health and medical capacity.

\section{The Material Reviewed}

There are some collections of information and material being set up on the internet (see website listings and references in Section 11 for examples). The documentation available is dynamic and being further developed. In most cases, it represents the initial framing up of thinking based on considering the COVID19 requirements and projecting them onto practices, procedures and protocols that have been applied and practiced in the past. We reviewed $60+$ items including 19 websites provided by participants of our survey (Section 8), and three websites that brought together publicly available material. These materials came from a range of government, non-government and private sector actors in fire management. 
The material reviewed indicate that adjustments needed under COVID-19 can be considered as strategic or operational. They can then be further characterized as being relevant to Risk Reduction/Prevention; Readiness; Response/Suppression and Recovery. After some broader description, these headings are used to collate ideas, suggestions and implications.

- The strategic level includes the framing up of fire management options that 1 ) are no longer viable under COVID-19; 2) can be applied but are constrained in some way; and 3) will have to be prioritized. There may also be options that are now included, though they may have not been selected or seriously considered in the past.

- The operational aspects include those changes that have implications for fire management staff, their transport, equipment use and handling and transfer, mobilization and demobilization, adjusting techniques and practices and so on.

In both strategic and operational aspects, there is minimal experience to date of the limitations of adhering to COVID-19 requirements, with very few examples to date of feedback from the field (but noting that this is starting to happen).

\section{Fire Management Considerations}

Firefighters and local communities deal with wildfire annually. This year, they must also take steps to reduce exposure to the COVID-19 pandemic and implement COVID-19 government regulations into their fire strategies and operations. Workplace cleanliness and hygiene is critical and this includes the full range of fire management 'workplaces': coordination and command, attendance and dispatch, field briefing areas, field operations, transportation, support and logistics. The strong focus on COVID-19 must be maintained and will have impacts on fire management, requiring changes in the mode of working, operations and strategy.

The requirements to reduce risk of exposure to COVID-19 will likely constrain fire management capacity (numbers, types, response times, resources for large scale fires and so on) and that will likely increase risk of fires becoming larger and potentially damaging.

Personnel may be concerned about contracting COVID-19 and spreading it to other firefighters and the public as they travel between incidents, and may be worried about bringing the virus home to their families. This may further reduce the availability of staff and/or reduce their flexibility to travel and work away from their local area.

\subsection{Approaches to Fire Suppression}

The approaches taken for fire management vary between countries, jurisdictions and agencies. This is expected and reflects the fire management needs, systems and history in each case.

In some countries like Australia, the model is that generally staff reside at home and attend the fire via a collection point (office, depot, control centre) and then return at the end of a shift via the collection point to home. This creates the potential for infection from and/or to the home.

There are countries like the USA where fire suppression staff are housed in large camps with communal facilities, catering and logistics support that results in large numbers of people gathered in one location and moving through it daily. This likely increases the risk of infection between fire staff, as opposed to the situation where people sleep and rest at home. 
There are also very localized arrangements, like in some developing countries, that can be informal, where the community undertakes fire management, including suppression, in a way that is locally organized and led. The risk of infection is from or to the home and among the community engaged in fire suppression.

\subsection{COVID-19 Limitations for Fire Management}

Under the requirements for COVID-19 there will be a number of limitations for fire management. These include the following that have been extracted from the material reviewed. They are edited and somewhat generalized:

- Ensuring people's safety from fire and ensuring firefighter safety from COVID-19:

- The need to combine "social distancing" with fire management and fire suppression tasks. Both social distancing and fire management are essential to ensure people's safety. The extent to which they can be accommodated simultaneously is untested.

- Fire suppression agencies may experience a COVID-19 outbreak during fire response. The impact of this on fire suppression effectiveness and capacity, as well as on individual (and family/community) terms, could be significant.

- Evacuation planning. At times evacuations are ordered due to wildfire, but people also choose to evacuate themselves. If fire suppression resources are limited, then evacuations may occur earlier and be greater in scale.

- Ensuring people's safety in emergency shelters. The use of emergency shelter locations in case of community evacuations can result in large numbers of people that will not be able to meet social distancing requirements.

\section{Initial Guidance for Fire Management under COVID-19}

Overall the objectives that were presented in the materials we reviewed seek to prioritise efforts to protect fire management staff from COVID-19 exposure and transmission. As indicated in Section 4, these materials came from a range of government, non-government and private sector actors from around the world.

Note that we, the authors of this Brief, do not have medical expertise and cannot judge the soundness of these measures. Further, this is likely incomplete; more will be added in time and we release this now to allow fire managers around the world to be aware of this initial guidance.

\subsection{General Principles and Approaches}

\subsubsection{Keeping Firefighters Healthy to Reduce Risk of Contracting COVID-19}

- The fire season is inherently taxing on firefighters fatigue levels and immune systems, which may compromise their ability to fight off COVID-19 if exposed to it. Early studies suggest that air pollution may increase the impact of COVID-19 and that wildland firefighters may be more vulnerable to COVID-19 (link) because of repeated exposure to wildland fire smoke and fine particles in that smoke. Building more rest and recovery time into the season or utilizing different suppression tactics that reduce exhaustion and exposure to smoke may allow firefighters to remain healthy longer.

- Procedures must be in place to enhance cleaning and disinfecting vehicles, aircraft, common areas, bunkhouses and all high-touch areas. 
- Food service must focus on minimizing the handling of shared food and items by eliminating all shared food containers from dining areas and requiring kitchen staff to distribute food rather than field staff to gather together and collect it.

\subsubsection{Social Distancing}

- Access to all worksites must be controlled and restricted to essential personnel only. For this purpose, create and communicate clear procedures for defining 'essential personnel.' These would be based on considering who is needed in-person and which activities can be done remotely via phone, virtual meeting or other communication platform.

- Where practical, consider the use of virtual briefings to minimize contact. Emphasise use of radio, email, virtual meeting platforms or other similar modalities for briefings and materials. If not feasible then only team leaders should attend briefings.

- Command and general staff do not need to be in the same place as the firefighters.

- If essential, training will occur in small well-spaced groups and online wherever possible to reduce exposure.

- Reduction of work hours or additional (cleaning) staff may be needed to allow time for disinfecting and hygiene, which may require additional staff.

- Identify the positions and tasks of incident management team staff members that can be carried out by working remotely via phone, virtual meeting or other communication platform.

\subsubsection{Pre-Incident Screening}

- All personnel need to be tested for COVID-19 to establish a baseline of health information. Recent (travel) history should be recorded to determine the potential exposure risk to COVID-19.

- Restrict use of personnel who fall into an at-risk group such as people 65 years and older, and people of all ages with underlying medical conditions (such as people who are immunocompromised, with diabetes, with chronic lung disease or moderate to severe asthma, etc).

\subsubsection{While on Assignment}

- Conduct daily COVID-19 assessments to ensure the safety of staff.

- Encourage those who may be symptomatic to report to their supervisor as soon as possible. The procedures in place if someone gets ill, medical costs, salary continuity and other impacts need to be set out and communicated clearly in advance.

- Have isolation facilities and a plan in place.

- Have in place a specific ambulance and medical tent just for suspected cases and/or incidentwithin-an-incident planning and operations in case of a COVID-19 outbreak.

- Have a medical staff assigned to the incident dedicated to providing guidance and oversight of COVID-19 specific response and mitigation measures.

\subsubsection{Post-Assignment}

- Provide COVID-19 screening at the end of each fire assignment or before return to home. 


\subsubsection{Transportation and Aviation}

- Conduct only essential flights, and limit personnel on board to essential resources only.

- For staff that need to travel to the fireground, avoid, as practicable, commercial air travel and drive or charter an aircraft for crew transport to and from the home base or alternate work location.

- Where possible, ensure a crew will stay as a crew (social distancing between units) when being transported by vehicle or helicopter.

- Keep the front passenger seat of the helicopter vacant if not operationally required.

- Require face masks or shields to be worn inside vehicles if there is more than one person in a vehicle and physical distancing cannot be maintained. In some cases, this requirement may be modified for crews operating as independent units and controlling exposure, as the masks/shields have been found to constrict breathing (affecting rest and recuperation) and limit some visibility during vehicle operation.

- Implement control procedures to ensure vehicle sanitization and hand washing/sanitizing supplies are available at all times, that sanitization is completed, and that there is compliance with procedures.

\subsection{Fire Risk Reduction}

As the summer arrives, most risk reduction activities may have already been completed or are no longer operationally possible. These may include fuel reduction (e.g. by burning, grazing and other means) and establishing and maintaining containment features (e.g. fuel breaks). It was noted in the materials that COVID-19 has negatively affected public events for annual wildfire mitigation and prevention activities. The efforts to reduce the risk of ignitions is one focus that can be made and the materials reviewed included the following:

- People are the cause of nearly all fires. Fewer human-caused fires will not only help protect communities from wildfire, but will also preserve firefighting resources and help slow the spread of COVID-19 by reducing the need to move firefighters to fires. Consequently, a focused communication effort (using means that satisfy social distancing) to reinforce care with fire use may significantly assist in reducing human caused ignitions.

- Additionally, enhanced wildfire prevention actions to reduce wildfire risk could be applied, such as open burning restrictions, restrictions (or banning) use of fire in recreation (campfires, barbeques, cooking), and increased attention to vegetation management adjacent to infrastructure that can be an ignition source (railway lines, power lines, access to forests and protected areas) in an effort to reduce human caused ignitions.

\subsection{Readiness for Fires}

Readiness for fires includes climate, weather monitoring and prediction; a fire danger rating system; public notification means for fire danger rating and warnings; detection/suppression needs assessments; fire detection; suppression and communications resources; fire training systems; and, means of fire access. In most cases the readiness tasks will be feasible under COVID-19 as they involve routine operation of existing systems (fire danger rating) and often do not require groups of people (fire detection). One aspect that may be compromised is training. 
Readiness training is part of wildfire season preparation and includes certification and qualifications to ensure a viable resource response. Education and physical testing are part of this annual process. The materials reviewed noted that social distancing directives and cancellation of courses have compromised this system of group training. In some cases it was noted that qualifications had been extended where requalification had not been possible due to COVID-19 restrictions.

\subsection{Response to Fires}

Fire suppression involves a range of activities, including detection and reporting, first response, containment and control, mop up and patrol, and command and control. Summarizing the materials reviewed, the proposed adjustments in procedures for fire suppression include:

- Prioritize the use of local suppression resources with the predominant strategy being rapid containment. With the increased infection risk associated with travel, use the closest agency resources first.

- Wherever possible, use aggressive initial attack supported by available aerial means, to extinguish wildfires quickly and minimize the need to bring large numbers of firefighters together.

- One caveat was noted with this approach: aggressive initial attack may incline fire suppression staff to accept undue personal risk from fire-related injury in an attempt to keep the fire small.

- To help personnel remain isolated, allocate only the amount and types of fire suppression resources necessary to manage the fire.

- Commit resources only when there is a reasonable expectation of success in protecting life and critical property and infrastructure.

- Utilize more managed fire as a tactic in suppression. This has the potential to reduce the need for suppression resources.

- Modify fire suppression tactics to use existing features (e.g., roads, rivers) as well as point protection to limit the numbers of personnel needed in creating fireline.

- Utilize more heavy equipment for creating fireline and reduce the crew resources needed.

- Reduce the amount of mop-up used; let fires go out naturally, accepting the added risk of flaring up and reignition.

- For larger fires that aren't controlled during initial attack, the reliance on large fire camps gathering and supporting hundreds of firefighters will not be safe. Where fire camps have been used, initial survey respondents indicate that large fire camps will not be the norm under COVID19. They suggest fire suppression efforts could be in small groups and dispersed into isolated camps for better social distancing and safety from spread of COVID-19.

When using managed fire and using existing features, an additional consideration is the potential for increased area burned over a longer period of time; this may increase smoke exposure to the public (and therefore potentially increased susceptibility to COVID-19), a risk that should be mitigated and balanced against other exposure risks. 


\subsection{Protecting Remote Communities from Infection}

It has been identified that there are likely to be specific issues for vulnerable communities, particularly indigenous communities that are particularly susceptible to infection such as recognised in Alaska and in parts of South America. Firefighters would in the past set up command posts in the communities themselves, to take advantage of infrastructure and to provide economic benefits. This comes with the risk of entering and infecting remote/isolated communities and there is discussion of eliminating that practice altogether (setting up command in more remote areas). The considerations around these and similar types of vulnerabilities will need to be a focus for evaluation and monitoring to reduce the risk to firefighters from the public, but importantly to protect communities from firefighters who might be carrying COVID19.

\section{Lessons Learned so Far}

There has been some reporting on lessons learned and discussion of experiences during fire suppression already in Western Europe and North America. These initial reflections include:

- To mitigate for social distancing in vehicles, travel to the incident was conducted in more vehicles than are typically used.

- Prior to travel, intentional discussions with module leaders were held concerning continuing social distancing and sanitation measures as much as possible.

- A crew member was not assigned because of precautionary quarantine due to potential COVID19 exposure during the previous week.

- Social distancing is tough in stressful situations. In one example, a crew discussed social distancing before leaving for the fire to try and abide by the COVID-19 guidelines. Upon arrival, multiple structures and vehicles were burning and instincts to protect life and property took over.

- Maintaining distance is difficult during a public evacuation when property owners and landowners are panicked and looking for answers and guidance.

- When working with multiple agencies, their COVID-19 mitigation measures and messages varied widely and this lack of consistency made them hard to enforce or maintain.

- Briefings with large numbers of people are hard to conduct in the field while maintaining the recommended physical distancing. Microphones or platforms are not usually available in initial attack, which made it difficult to voice critical information to multiple crews.

- In one example, once dispatched, four vehicles with eight firefighters were used. Upon arrival, the parking and safety areas for vehicles was minimal, causing clustering of them. Upon arrival, personnel jumped into different trucks and engines to engage the fire-creating more "contaminated" surfaces.

- Those who did wear masks seemed to be touching their faces more to adjust masks.

- It was difficult to keep equipment sanitized throughout an incident. (Examples: truck radios, hand tools, chainsaws, steering wheels, compartment doors, etc.)

- Individuals who wore masks experienced a harder time communicating. 


\section{Preliminary Survey Results}

\subsection{About the Survey: Fire Management Under COVID-19}

The survey Fire Management under COVID-19 (link) was circulated to the international fire community on 17 April 2020, aimed at wildland fire management professionals around the world in fire, land management and related agencies that fill policy, management, incident command or field roles and supporting specialists.

The goal of the survey is to clarify the implications of COVID-19 restrictions on wildland fire management, map current thinking and collate any plans, protocols or procedures to prepare an initial list for consideration by those with the responsibility for fire management. We here defined fire management as including the full disaster management cycle from risk reduction (prevention, mitigation) and readiness (preparedness, preparation), to response (suppression), recovery (restoration, rehabilitation) and review. In addition several descriptive questions were asked about the country of origin, type of agency, level of jurisdiction, and type of fire the respondents worked at.

The survey targets wildland fire management professionals around the world in fire, land management and related agencies that fill policy, management, incident command or field roles and supporting specialists. Its rationale is that if the agencies in countries work in isolation or internally on COVID-19 they may take longer to get to the same set of ideas, overlook some ideas and/or prioritise less efficiently.

\subsection{Type of Responses to Date}

As of 4 May 2020, 17 days after opening the survey, 348 people had filled it out from around the world. The median time taken between starting and ending the survey was 10 minutes. The survey was fully anonymous so no names of agencies or individuals were collected. As the survey remains open until 15 May, we share here only a description of the type of respondents that have filled out the survey so far.

Most respondents to date are solely active in wildland fire ( 70\%), with $12 \%$ being active in both wildland fire and structural fire. $18 \%$ did not answer this question.

Geographically, respondents were based in 26 countries (Fig. 1). A major part, 160 respondents, or $45 \%$ of the total, was based in the USA. On a long distance, this was followed by Spain, Italy, South Africa and Australia being represented with between 13 and 21 responses, and Portugal and Greece with 9 and 6

responses. One to a few responses each were from Indonesia and India, Latin American countries (Uruguay, Bahamas, Brazil, Ecuador), central and eastern Europe (Switzerland, Republic of North Macedonia, Georgia, Latvia, Romania), Northwestern Europe (Netherlands, Sweden, United Kingdom), France, Israel and the Russian Federation. It is great to see such varied response also from countries not typically associated with wildland fire.

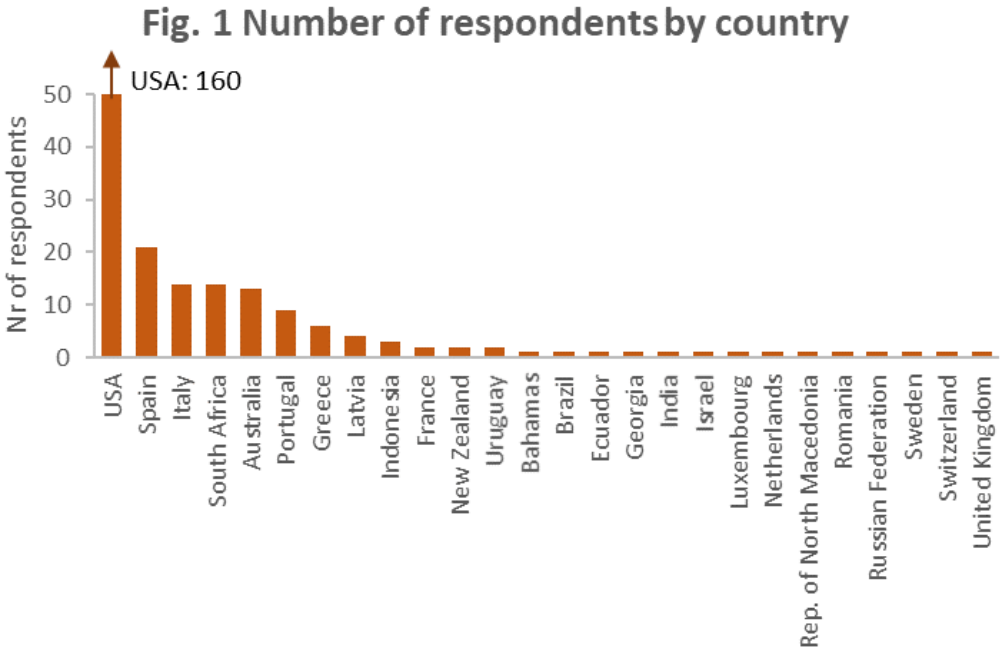


Respondents worked mostly at national and province/state agencies (Fig. 2) in government or the private sector (Fig. 3). Five responses were from tribal agencies (Fig. 2). The respondents' primary and secondary functions are predominantly in land management, fire management and emergency services (Fig. 4-5). The data show a good spread across the size of the fire management and suppression staff (Fig. 6-7), in which organizations with $<10$ fire management staff represent a third of responses (Fig. 6).

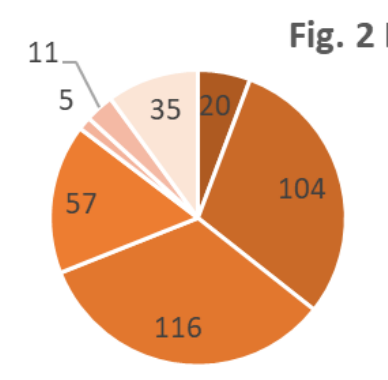

ig. 2 Level of jurisdiction

Fig. 3 Type of organization

- International
- National
- Province/state
- Local
- Tribal
- Other
- No data

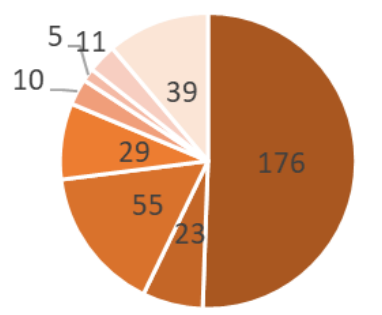

- Government

- Civil protection

- Private

- NGO

- $\mathrm{CBO}$

Multilateral

- Other

No data

Fig. 4 Primary function

Fig. 5 Secondary function

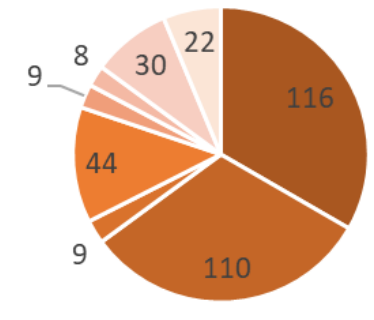

- Land management

- Fire management

- Structural fire

- Emergency services

Specialist services

- Support service

Other

No data

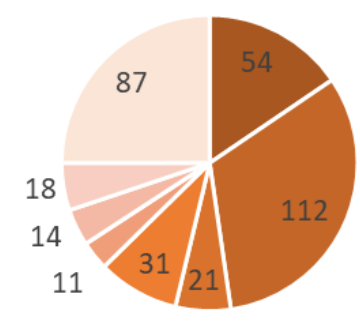

- Land management

- Fire management

- Structural fire

- Emergency services

- Specialist services

- Support service

- Other

No data

Fig. $6 \mathrm{Nr}$ of fire management staff

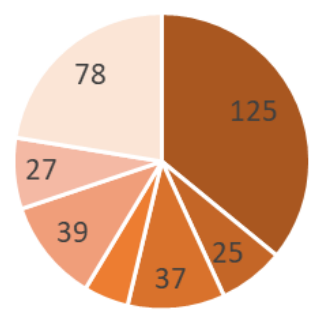

$$
\begin{aligned}
& =1-10 \\
& =11-20 \\
& =21-50 \\
& =51-100 \\
& =101-500 \\
& =500+ \\
& =\text { No data }
\end{aligned}
$$

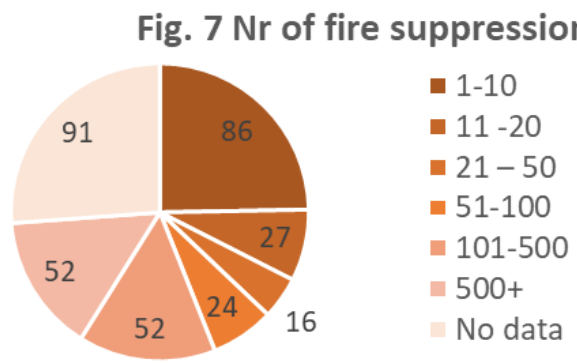

17

Preliminary overview of survey respondents as of 4 May 2020. Total $n=348$. Missing data (absence of response) is listed as 'no data'. Values listed in each graph represent the absolute number of cases in each category.

The respondents submitted 14 documents for review as well as 19 website URLs, which we analysed for this Brief along with materials sourced through searches and provided by colleagues (Section 4). From the responses, it is clear that the impact of COVID-19 on fire management is being recognized and considered. 


\subsection{Next Steps: Survey Still Open, Findings to be Published Late May 2020}

We extended the survey response deadline to 15 May 2020, and very much welcome additional responses, particularly from individuals, agencies or groups that are currently underrepresented in the survey. Given the current distribution of respondents, we particularly welcome additional survey respondents from the global fire community, in Latin America, Australasia, Africa, Europe and the Middle East, as well as from Canada. In the US and elsewhere, we additionally welcome views from tribal agencies that are currently underrepresented in the survey responses. We finally not only welcome responses from traditionally fire-prone regions and countries but also from non-traditionally fire-prone regions like temperate climate zones. Our second Brief (planned for publication on or around 25 May) will detail the survey results, the perceived impact of COVID-19 on fire management, and strategies shared to deal with these impacts.

\section{Conclusion and Outlook}

The requirements put in place to deal with COVID-19 in most countries include some level of restriction on movement, business and social interaction, and in many countries this has taken the form of a "lockdown". These measures for COVID-19 must be considered in the context of fire management procedures, standards and approaches as the Northern Hemisphere enters the summer and the fire season.

This Brief was prepared based on materials sourced from government agencies, NGOs and the private sector with the intention of clarifying the implications of COVID-19 restrictions on wildland fire management, map current thinking and raise awareness of the issue.

The strong investment in assessing, thinking and planning indicates that the regular processes of risk reduction/prevention, readiness to suppress fires and, in particular, fire suppression will be carried out with complicating requirements and additional limitations due to COVID-19. These factors collectively will potentially influence strategic fire management options, fire suppression operations and the means and modes of engaging with the public before, during and after wildfires.

The strategies and tactics have barely begun to be 'refined' or 'changed' through experience. As the summer progresses and operations are conducted, we plan to collect further insights, changes and adjustments to be incorporated, summarized and circulated as appropriate.

For the near term, our survey (Section 8) will stay open until 15 May 2020 and we plan to publish its results in a second Brief on or around 25 May 2020. We very much welcome additional responses to the survey. More information can be found here.

\section{Acknowledgements}

We sincerely thank all the colleagues from the international wildland fire community who helped by sending us publications, work in progress and other information, and by distributing the survey within their professional networks. 


\section{Reference List}

Set out below are examples of the materials and some websites that contain materials on COVID-19.

- National Wildfire Coordinating Group (USA/Canada) - Infectious Disease Guidance for Wildland Fire Incidents, Emergency Medical Committee - https://www.nwcg.gov/committees/emergencymedical-committee/infectious-disease-guidance

- US Department of Interior Office of Wildland Fire - https://www.doi.gov/wildlandfire/wildfirescovid-19

- What Does Fire Season Look Like Amidst the COVID-19 Pandemic https://fireecology.org/resources/Documents/HPIOL COVID-19.pdf

- Statement issued April 1, 2020 by the Western Fire Chiefs Association (https://wfca.com) https://www.iawfonline.org/article/2020-04-wildfires-pandemic-whats-ahead-wfca/

- Wildland Fire Response Plan COVID-19 Pandemic Eastern Geographic Area (US) April 2020

- Wildland Fire Response Plan COVID-19 Pandemic Southern Geographic Area (US) April 2020

- Wildland Fire Response Plan COVID-19 Pandemic Rocky Mountain Geographic Area (US) April 2020

- Wildland Fire Response Plan COVID-19 Pandemic Southwest Geographic Area (US) April 2020

- Dean Ferreira, Standard Operating Procedure for COVID-19 NCC Environmental Services (Pty) LTD Republic of South Africa

- Virginia Department of Forestry (USA) - http://www.dof.virginia.gov/covid/index.htm

- Association of Fire Ecology (USA) - https://fireecology.org/COVID-19

- Matthew P Thompson, Erin Belval, Jude Bayham - Quantitative Risk Assessment (QRA) of COVID-19 Outbreak in Fire Camp 